\title{
A parallel distributed compensation approach to fuzzy control of spacecraft combined attitude and sun tracking
}

\author{
Yew-Chung Chak*, Renuganth Varatharajoo ${ }^{\dagger}$ \\ Department of Aerospace Engineering, Faculty of Engineering, Universiti Putra Malaysia, 43400 Serdang, Selangor, Malaysia \\ *Corresponding author E-mail: samchak11@gmail.com, ${ }^{\dagger}$ renu99@gmx.de
}

\begin{abstract}
A spacecraft combined attitude and sun tracking system (CASTS) is a synergized system in which solar array drive assemblies are used as sun trackers and simultaneously as attitude control actuators. This paper, a continuous research on CASTS, addresses its attitude control problem. The kinematics and dynamics equations of a rigid spacecraft attitude motion is inherently nonlinear. In the att itude regulation problem, the attitude motion can be treated as a simple linear system for a constrained range of operating conditions, but it has impacts on the accuracy of the linear model when a model-based controller is implemented. Naturally, this is a compromise between simplicity and accuracy that all design engineers have to face. In this paper, we present a systematic approach to improve the accuracy while preserving the model as linear as possible, by deriving a quasi-linear approximation of a nonlinear spacecraft attitude motion. The quasilinear approximation is based on the framework of the Takagi-Sugeno (T-S) fuzzy model. If a spacecraft can be modeled in the form of a rule-based T-S fuzzy system that acts as an interpolator between linear state-space systems, an approach called parallel distributed control (PDC) can be used to stabilize the attitude motion. The design philosophy of PDC is to create a simple fuzzy controller, where each rule's consequent is a control law designed to stabilize the linear system in the corresponding consequent of the spacecraft T-S fuzzy system. Numerical results validate that the attitude and sun-tracking performances are achievable using the proposed PDC strategy.
\end{abstract}

Keywords: attitude control; fuzzy control; parallel distributed compensation; spacecraft; Takagi-Sugeno fuzzy model.

\section{Introduction}

One of the latest satellites added to the list of spacecraft failures is the Zuma satellite, a secretive United States government satellite that was launched by SpaceX on 8 January 2018 [1]. A total loss of spacecraft can cost the government billions of dollars. A common cause of spacecraft failure is the reaction wheel faults on one of the rotational axis and, leaving the effects of external disturbances uncompensated, leading to spacecraft tumbling about the three axes, roll, pitch, and yaw [2].

An expansive way to recover from a tumbling state is to use the reaction thrusters. To save on the propellant consumption, the Solar Array Drive Assembly (SADA), a type of momentum exchange device for "orientating" the solar arrays directly at the Sun for maximum conversion of sunlight, can be exploited for attitude controls. The idea of SADA is pioneered by Varatharajoo based on the innovations of synergistic systems for small satellites [3-4]. For a bi-wing three-axis stabilized spacecraft, a differential torque can be generated when the two solar arrays are rotated at different angular rates. This attitude control architecture is known as the Combined Attitude and Sun Tracking System (CASTS) [5].

The previous study on the CASTS was based on linearized attitude dynamics on the pitch axis. The proposed attitude control scheme in this paper deals with the nonlinear multivariable control problem of attitude motion, with the objective to stabilize the attitude in the presence of disturbance and to track the Sun simultaneously. The remaining part of this paper is organized as follows. The CASTS control architecture is described in the Section 2. The fuzzy modelling of a rigid spacecraft and control problem formu- lation are developed in the Section 3. The fuzzy parallel distributed controller (PDC) is designed in the Section 4. The stability condition for asymptotic stability is provided in Section 5. The simulation results are discussed in the Section 6 , followed by the conclusions given in the last section.

\section{CAST system architecture}

As shown in Figure 1, the SADA is the critical component of the CASTS control architecture, which serves as a torque generator between the solar array and the main hub. On a typical bi-wing three-axis stabilized Earth satellites, SADAs and the solar arrays are mounted on the pitch axis.

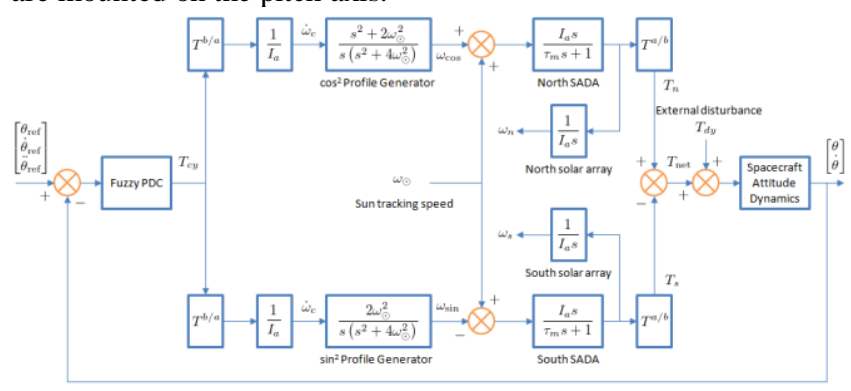

Fig. 1: CASTS control architecture.

\subsection{Sun Tracking Control}

For Sun tracking in a single plane, the solar arrays is rotated by the SADAs. Since the SADA uses a brushless DC motor, it can be 
modelled as first-order system after neglecting the electrical dynamics

$$
\frac{\omega_{m}(s)}{\omega_{c}(s)}=\frac{1}{\tau_{m} s+1}
$$

where $\omega_{m}$ is the angular velocity of the motor, $\omega_{c}$ is the angular velocity commanded by the control law, and $\tau_{m}$ is a time-constant of the motor. The aim is to drive the error between $\omega_{m}$ and $\omega_{c}$ to zero.

\subsection{Attitude Manoeuvre Control}

In Figure 1 the desired control torque, $T_{c y}$ for controlling the pitch attitude and tracking the Sun simultaneously, can be generated by rotating the north and the south solar array paddles at different angular velocities about the pitch axis of the spacecraft while maintaining a low incident angle with relative to the Sun axis to ensure a maximum power-point tracking.

By Newton's second law for rotational motion, $T=I \dot{\omega}$, and the Pythagorean trigonometric identity $\cos ^{2}(\theta)+\sin ^{2}(\theta)=1$, the net torque, $T_{\text {net }}(s)$, can be written in Laplace transform as a result from the differential torques generated by both SADA actuators

$T_{\text {net }}(s)=T_{n}(s)-T_{s}(s)=\left(\frac{1}{\tau_{m} s+1}\right) T_{c y}(s)$

where $T_{c y}$ is the control torque commanded by the fuzzy PDC control law. The actual torques produced by the rotations of the north and south wings are denoted by $T_{n}$ and $T_{s}$, respectively [6]

$$
\begin{aligned}
& T_{n}(s)=\frac{I_{a} s}{\tau_{m} s+1}[\omega_{\odot}+\underbrace{\frac{s^{2}+2 \omega_{\odot}^{2}}{s\left(s^{2}+4 \omega_{\odot}^{2}\right)}\left(\omega_{c} s\right)}_{\omega_{\cos }}] \\
& T_{s}(s)=\frac{I_{a} s}{\tau_{m} s+1}[\omega_{\odot}-\underbrace{\frac{2 \omega_{\odot}^{2}}{s\left(s^{2}+4 \omega_{\odot}^{2}\right)}\left(\omega_{c} s\right)}_{\omega_{\text {sin }}}]
\end{aligned}
$$

where $I_{a}$ is the moment of inertia about the pitch axis of each solar array, and $\omega_{\odot}$ is the reference Sun tracking speed (assumed to be the same as the orbital velocity of the satellite).

\section{Fuzzy modelling of a rigid spacecraft}

In this section, the attitude dynamics of a rigid spacecraft is modelled by means of Takagi-Sugeno (T-S) fuzzy model.

\subsection{Takagi-Sugeno (T-S) fuzzy model}

The fuzzy model proposed by Takagi and Sugeno [7], uses a set of reasoning rules in the form of If-Then linguistic descriptions to represent the local linear input-output relations based on the derivations from the given nonlinear system equations. The advantage of using a Takagi-Sugeno fuzzy model allows the local dynamics of each fuzzy implication to be expressed by a linear state-space representation. Consider a $\mathrm{T}-\mathrm{S}$ fuzzy system with $R$ rules of the form $[8,9]$ :

$$
R^{i} \quad \text { If } z_{1} \text { is } M_{1}^{K} \wedge \ldots \wedge z_{n} \text { is } M_{n}^{L}, \quad \text { Then } \dot{\boldsymbol{x}}^{i}=\mathbf{A}_{i} \boldsymbol{x}+\mathbf{B}_{i} \boldsymbol{u}
$$

where $z_{n}(t)$ is the premise variable which may be functions of the state vector, $\boldsymbol{x}(t) \in \mathbb{R}^{\boldsymbol{n}}$ that varies continuously with time $t$; $\boldsymbol{u}(t) \in \mathbb{R}^{\boldsymbol{m}}$ is the input vector; $M_{n}^{L}$ is the fuzzy set; $\mathbf{A}_{i} \in \mathbb{R}^{\boldsymbol{n} \times \boldsymbol{n}}$ is the state matrix and $\mathbf{B}_{i} \in \mathbb{R}^{\boldsymbol{n} \times \boldsymbol{m}}$ is the input matrix. The state-space representation, $\mathbf{A}_{i} \boldsymbol{x}+\mathbf{B}_{i} \boldsymbol{u}$ at each linear consequent rule is called a 'subsystem.' The output of the fuzzy system is a time-varying system described by the defuzzification procedure:
$\dot{\boldsymbol{x}}(t)=\frac{\sum_{i=1}^{R}\left\{\mu_{i}(z(t))\left[\mathbf{A}_{i} \boldsymbol{x}(t)+\mathbf{B}_{i} \boldsymbol{u}(t)\right]\right\}}{\sum_{i=1}^{R} \mu_{i}(z(t))}$

or

$\dot{\boldsymbol{x}}(t)=\mathbf{A}(t) \cdot \boldsymbol{x}(t)+\mathbf{B}(t) \cdot \boldsymbol{u}(t)$

where the time-varying state matrix is $\mathbf{A}(t)=\xi_{1}(t) \mathbf{A}_{1}+$ $\xi_{2}(t) \mathbf{A}_{2}+\cdots+\xi_{R}(t) \mathbf{A}_{R}$, the time-varying input matrix is $\mathbf{B}(t)=\xi_{1}(t) \mathbf{B}_{1}+\xi_{2}(t) \mathbf{B}_{2}+\cdots+\xi_{R}(t) \mathbf{B}_{R}, \quad$ and $\xi_{i}(t), i=$ $1, \ldots, R$ are the fuzzy basis function described by:

$\xi_{i}(t)=\frac{\mu_{i}(z(t))}{\sum_{i=1}^{R} \mu_{i}(z(t))}, \quad i=1, \ldots, R$.

\subsection{Construction of fuzzy model}

Consider the following rigid spacecraft system which has the nonlinear mathematical model in Euler angles form:

$\left[\begin{array}{c}\dot{\phi} \\ \dot{\theta} \\ \dot{\dot{x}} \\ \dot{\omega}_{x} \\ \dot{\omega}_{y} \\ \dot{\omega}_{z}\end{array}\right]=\left[\begin{array}{cccccc}0 & 0 & 0 & 1 & s_{\phi} \tan \theta & c_{\phi} \tan \theta \\ 0 & 0 & 0 & 0 & c_{\phi} & -s_{\phi} \\ 0 & 0 & 0 & 0 & s_{\phi} \sec \theta & c_{\phi} \sec \theta \\ 0 & 0 & 0 & 0 & 0 & \left(\frac{I_{y}-I_{z}}{I_{x}}\right) \omega_{y} \\ 0 & 0 & 0 & \left(\frac{I_{z}-I_{x}}{I_{y}}\right) \omega_{z} & 0 & 0 \\ 0 & 0 & 0 & 0 & \left(\frac{I_{x}-I_{y}}{I_{z}}\right) \omega_{x} & 0\end{array}\right]\left[\begin{array}{c}\phi \\ \theta \\ \psi \\ \omega_{x} \\ \omega_{y} \\ \omega_{z}\end{array}\right]+\left[\begin{array}{ccc}0 & 0 & 0 \\ 0 & 0 & 0 \\ 0 & 0 & 0 \\ \frac{1}{I_{x}} & 0 & 0 \\ 0 & \frac{1}{I_{y}} & 0 \\ 0 & 0 & \frac{1}{I_{z}}\end{array}\right]\left[\begin{array}{c}u_{x} \\ u_{y} \\ u_{z}\end{array}\right](9)$

where $s_{\phi}=\sin \phi, c_{\phi}=\cos \phi,\left\{I_{x}, I_{y}, I_{z}\right\}$ are the principal moments of inertia, and $\left\{u_{x}, u_{y}, u_{z}\right\}$ are the control inputs to the system to be designed via the parallel distributed compensation approach in Section 4.

If small angles and $I_{x}=I_{z}$ are assumed, then the model can be reduced to a quasi-linear system:

$\left[\begin{array}{c}\dot{\phi} \\ \dot{\theta} \\ \dot{\psi} \\ \dot{\omega}_{x} \\ \dot{\omega}_{y} \\ \dot{\omega}_{z}\end{array}\right]=\left[\begin{array}{cccccc}0 & 0 & 0 & 1 & 0 & 0 \\ 0 & 0 & 0 & 0 & 1 & 0 \\ 0 & 0 & 0 & 0 & 0 & 1 \\ 0 & 0 & 0 & 0 & 0 & \left(\frac{I_{y}-I_{z}}{I_{x}}\right) \dot{\theta} \\ 0 & 0 & 0 & 0 & 0 & 0 \\ 0 & 0 & 0 & 0 & \left(\frac{I_{x}-I_{y}}{I_{z}}\right) \dot{\phi} & 0\end{array}\right]\left[\begin{array}{c}\phi \\ \theta \\ \psi \\ \dot{\phi} \\ \dot{\theta} \\ \dot{\psi}\end{array}\right]+\left[\begin{array}{ccc}0 & 0 & 0 \\ 0 & 0 & 0 \\ 0 & 0 & 0 \\ I_{x}^{-1} & 0 & 0 \\ 0 & I_{y}^{-1} & 0 \\ 0 & 0 & I_{z}^{-1}\end{array}\right]\left[\begin{array}{l}u_{x} \\ u_{y} \\ u_{z}\end{array}\right]$

Defining $z_{1}=-\left(\frac{I_{y}-I_{z}}{I_{x}}\right) \dot{\theta}$ and $z_{2}=\left(\frac{I_{x}-I_{y}}{I_{z}}\right) \dot{\phi}$, then, the statespace system can be rewritten as

$\left[\begin{array}{c}\dot{\phi} \\ \dot{\theta} \\ \dot{\psi} \\ \dot{\omega}_{x} \\ \dot{\omega}_{y} \\ \dot{\omega}_{z}\end{array}\right]=\left[\begin{array}{cccccc}0 & 0 & 0 & 1 & 0 & 0 \\ 0 & 0 & 0 & 0 & 1 & 0 \\ 0 & 0 & 0 & 0 & 0 & 1 \\ 0 & 0 & 0 & 0 & 0 & -z_{1} \\ 0 & 0 & 0 & 0 & 0 & 0 \\ 0 & 0 & 0 & 0 & z_{2} & 0\end{array}\right]\left[\begin{array}{c}\phi \\ \theta \\ \psi \\ \dot{\phi} \\ \dot{\theta} \\ \dot{\psi}\end{array}\right]+\left[\begin{array}{ccc}0 & 0 & 0 \\ 0 & 0 & 0 \\ 0 & 0 & 0 \\ I_{x}^{-1} & 0 & 0 \\ 0 & I_{y}^{-1} & 0 \\ 0 & 0 & I_{z}^{-1}\end{array}\right]\left[\begin{array}{l}u_{x} \\ u_{y} \\ u_{z}\end{array}\right]$

In order to construct a $\mathrm{T}-\mathrm{S}$ fuzzy system that exactly represents the nonlinear system, Eq. (10), we consider an axisymmetric spacecraft with principal inertias $I_{x}=I_{z}=3102, I_{y}=564$, and assume the bounded domain $X$ is defined by $\dot{\phi} \in[-0.0002,0.0002]$, and $\dot{\theta} \in[-0.0002,0.0002]$. Then the minimum and maximum values of $z_{1}$ and $z_{2}$ can be obtained as follows:

$\min _{\chi} z_{1}=\min _{\chi} z_{2}=-\frac{9}{55000}, \quad \max _{\chi} z_{1}=\max _{\chi} z_{2}=\frac{9}{55000} .(12)$

The membership functions that describe the fuzzy sets on the $z_{1}$ and $z_{2}$ universes are given by [8]

$\mu^{\mathrm{NEG}}\left(z_{1}\right)=\frac{\max _{\chi} z_{1}-z_{1}}{\max _{\chi} z_{1}-\min _{\chi} z_{1}}, \quad \mu^{\mathrm{POS}}\left(z_{1}\right)=\frac{z_{1}-\min _{\chi} z_{1}}{\max _{\chi} z_{1}-\min _{\chi} z_{1}}$,

$\mu^{\mathrm{NEG}}\left(z_{2}\right)=\frac{\max _{x} z_{2}-z_{2}}{\max _{x} z_{2}-\min _{\chi} z_{2}}, \quad \mu^{\mathrm{POS}}\left(z_{2}\right)=\frac{z_{2}-\min _{\chi} z_{2}}{\max _{x} z_{2}-\min _{x} z_{2}}$. 

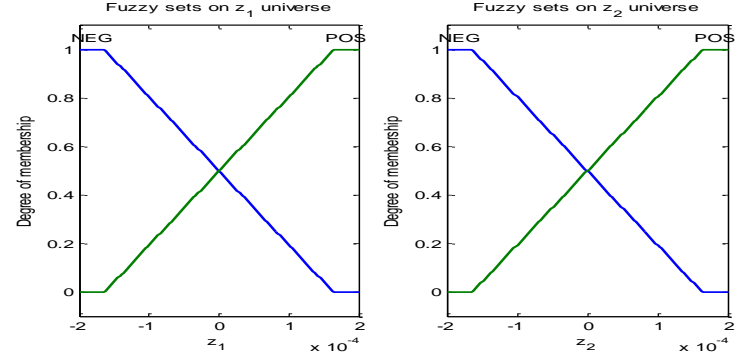

Fig. 2: Fuzzy sets on the universes of $z_{1}$ and $z_{2}$.

The rule base of the T-S fuzzy system is

- $\quad R^{1}$ : If $z_{1}$ is NEG and $z_{2}$ is NEG, then $\dot{\boldsymbol{x}}^{1}=\mathbf{A}_{1} \boldsymbol{x}+\mathbf{B} \boldsymbol{u}$,

- $\quad R^{2}$ : If $z_{1}$ is NEG and $z_{2}$ is POS, then $\dot{\boldsymbol{x}}^{2}=\mathbf{A}_{2} \boldsymbol{x}+\mathbf{B} \boldsymbol{u}$,

- $R^{3}$ : If $z_{1}$ is POS and $z_{2}$ is NEG, then $\dot{\boldsymbol{x}}^{3}=\mathbf{A}_{3} \boldsymbol{x}+\mathbf{B} \boldsymbol{u}$,

- $R^{4}$ : If $z_{1}$ is POS and $z_{2}$ is POS, then $\dot{\boldsymbol{x}}^{4}=\mathbf{A}_{4} \boldsymbol{x}+\mathbf{B} \boldsymbol{u}$, where

$\mathbf{A}_{1}=\left[\begin{array}{cccccc}0 & 0 & 0 & 1 & 0 & 0 \\ 0 & 0 & 0 & 0 & 1 & 0 \\ 0 & 0 & 0 & 0 & 0 & 1 \\ 0 & 0 & 0 & 0 & 0 & \frac{9}{55000} \\ 0 & 0 & 0 & 0 & 0 & 0 \\ 0 & 0 & 0 & 0 & -\frac{9}{55000} & 0\end{array}\right], \quad \mathbf{A}_{2}=\left[\begin{array}{cccccc}0 & 0 & 0 & 1 & 0 & 0 \\ 0 & 0 & 0 & 0 & 1 & 0 \\ 0 & 0 & 0 & 0 & 0 & 1 \\ 0 & 0 & 0 & 0 & 0 & \frac{9}{55000} \\ 0 & 0 & 0 & 0 & 0 & 0 \\ 0 & 0 & 0 & 0 & \frac{9}{55000} & 0\end{array}\right]$

$\mathbf{A}_{3}=\left[\begin{array}{cccccc}0 & 0 & 0 & 1 & 0 & 0 \\ 0 & 0 & 0 & 0 & 1 & 0 \\ 0 & 0 & 0 & 0 & 0 & 1 \\ 0 & 0 & 0 & 0 & 0 & -\frac{9}{55000} \\ 0 & 0 & 0 & 0 & 0 & 0 \\ 0 & 0 & 0 & 0 & -\frac{9}{55000} & 0\end{array}\right], \quad \mathbf{A}_{4}=\left[\begin{array}{cccccc}0 & 0 & 0 & 1 & 0 & 0 \\ 0 & 0 & 0 & 0 & 1 & 0 \\ 0 & 0 & 0 & 0 & 0 & 1 \\ 0 & 0 & 0 & 0 & 0 & -\frac{9}{55000} \\ 0 & 0 & 0 & 0 & 0 & 0 \\ 0 & 0 & 0 & 0 & \frac{9}{55000} & 0\end{array}\right]$

This T-S fuzzy model exactly represents the nonlinear system, Eq (10) in $\dot{\phi} \in[-0.0002,0.0002]$, and $\dot{\theta} \in[-0.0002,0.0002]$.

\section{Control system design}

This section presents the design procedure of parallel distributed compensation for the $\mathrm{T}-\mathrm{S}$ fuzzy model of spacecraft attitude dynamics.

\subsection{Parallel distributed compensation}

The design approach of parallel distributed compensation (PDC) was originally proposed by Kang and Sugeno [10] to provide a systematic procedure to design a set of linear controllers in form of a fuzzy system based on a given T-S fuzzy model. The design procedure was then improved by Tanaka and Sugeno [11] and the duo analysed the stability of the fuzzy PDC-based control system. Later, Wang, Tanaka, and Griffin [12] coined the design procedure 'parallel distributed compensation.'

In a way, the working principle of a fuzzy PDC is similar to the gain scheduling, because it continuously varies the linear controller gains depending on the state of the system process. In the PDC design, each control If-Then rule is constructed from the corresponding rule of a plant fuzzy model in Eq. (5), or more specifically each rule's consequent is a control law designed to stabilize the linear state-space, $\dot{\boldsymbol{x}}^{i}=\mathbf{A}_{i} \boldsymbol{x}+\mathbf{B}_{i} \boldsymbol{u}$ in the corresponding consequent of the plant fuzzy system. Hence, the rule premises of the fuzzy controller share the same fuzzy sets with the plant fuzzy system.

For a nonlinear system given by the T-S fuzzy model in Eq. (5), the pole placement technique can be used to design the PDC, which is another fuzzy system with R rules of the form $[8,9]$ :

$R^{i} \quad$ If $z_{1}$ is $M_{1}^{K} \wedge \ldots \wedge z_{n}$ is $M_{n}^{L}, \quad$ Then $\boldsymbol{u}^{i}=-\mathbf{K}_{i} \boldsymbol{x}$

where $\mathbf{K}_{i}$ are the local feedback gains to be determined to ensure that the eigenvalues of $\mathbf{A}_{i}-\mathbf{B}_{i} \mathbf{K}_{i}$ stays in the left half-plane of the complex plane.
The output of the fuzzy controller is

$\boldsymbol{u}(t)=-\frac{\sum_{i=1}^{R}\left\{\mu_{i}(z(t))\left[\mathbf{K}_{i} x(t)\right]\right\}}{\sum_{i=1}^{R} \mu_{i}(z(t))}=-\left[\sum_{i=1}^{R}\left\{\mathbf{K}_{i} \xi_{i}(t)\right\}\right] \boldsymbol{x}(t)$.

Remark 1: Although the feedback gains $\mathbf{K}_{i}$ in the fuzzy controller, Eq. (17) are designed using the pole placement technique to ensure local stability of $\mathbf{A}_{i}-\mathbf{B}_{i} \mathbf{K}_{i}$, the global design conditions are needed to guarantee the global stability and control performance, which will be provided in Section 5 .

\subsection{Stable controller design via pole placement}

Given the integrated state-space model (combined kinematics and dynamics)

$\dot{\boldsymbol{x}}^{i}=\mathbf{A}_{i} \boldsymbol{x}+\mathbf{B} \boldsymbol{u}$,

and the desired transient and stability performance

$\dot{\boldsymbol{x}}^{i}=-\Lambda \boldsymbol{x}$

where the stabilizing matrix $\boldsymbol{\Lambda}$ contains elements such as the desired damping term $2 \zeta \Omega$ and the stiffness term $\Omega^{2}$

$\boldsymbol{\Lambda}=\left[\begin{array}{cccccc}0 & 0 & 0 & 1 & 0 & 0 \\ 0 & 0 & 0 & 0 & 1 & 0 \\ 0 & 0 & 0 & 0 & 0 & 1 \\ \Omega_{x}^{2} & 0 & 0 & 2 \zeta_{x} \Omega_{x} & 0 & 0 \\ 0 & \Omega_{y}^{2} & 0 & 0 & 2 \zeta_{y} \Omega_{y} & 0 \\ 0 & 0 & \Omega_{z}^{2} & 0 & 0 & 2 \zeta_{z} \Omega_{z}\end{array}\right]$

then, by equating Eqs. (18) and (19), a state feedback controller can be computed:

$\boldsymbol{u}^{i}=\mathbf{B}^{-1}\left(-\boldsymbol{\Lambda}-\mathbf{A}_{i}\right) \boldsymbol{x}$

Thus, we can design a parallel distributed controller

$\boldsymbol{u}^{i}=-\left[\begin{array}{cccccc}k_{p x} & 0 & 0 & k_{d x} & 0 & -I_{x} z_{1} \\ 0 & k_{p y} & 0 & 0 & k_{d y} & 0 \\ 0 & 0 & k_{p z} & 0 & I_{z} z_{2} & k_{d z}\end{array}\right] \boldsymbol{x}=-\mathbf{K}_{i} \boldsymbol{x}$,

which places the closed-loop eigenvalues of each consequent of the spacecraft T-S fuzzy system at the desired poles $\lambda$ that satisfies the equation

$\Lambda x=\lambda x$.

The rule base of the fuzzy PDC controller is designed as

- $\quad R^{1}$ : If $z_{1}$ is NEG and $z_{2}$ is NEG, then $\boldsymbol{u}^{1}=-\mathbf{K}_{1} \boldsymbol{x}$,

- $\quad R^{2}$ : If $z_{1}$ is NEG and $z_{2}$ is POS, then $\boldsymbol{u}^{2}=-\mathbf{K}_{2} \boldsymbol{x}$,

- $R^{3}$ : If $z_{1}$ is POS and $z_{2}$ is NEG, then $\boldsymbol{u}^{3}=-\mathbf{K}_{3} \boldsymbol{x}$,

- $\quad R^{4}$ : If $z_{1}$ is POS and $z_{2}$ is POS, then $\boldsymbol{u}^{4}=-\mathbf{K}_{4} \boldsymbol{x}$, where

\begin{tabular}{|c|c|c|c|c|c|c|}
\hline \multirow{3}{*}{$\mathbf{K}_{1}=$} & {$[0.0124$} & 0 & 0 & 13 & 0 & $\min _{x}$ \\
\hline & 0 & 0.0023 & 0 & 0 & 2.053 & 0 \\
\hline & 0 & 0 & 0.0124 & 0 & $I_{z} \min _{x} z_{2}$ & 11.2913 \\
\hline & {$[0.0124$} & 0 & & 1.2913 & 0 & $-I_{x} \min _{x}$ \\
\hline \multirow[t]{3}{*}{$\mathbf{K}_{2}=$} & 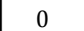 & 0.002 & 0 & & 2.0 & \\
\hline & 0 & 0 & 0.0124 & & $I_{z} \max _{x} z_{2}$ & 11.2913 \\
\hline & {$[0.0124$} & 0 & . & 2913 & 0 & $-I_{x} \max x$ \\
\hline \multirow[t]{3}{*}{$\mathbf{K}_{3}=$} & 0 & 0.0023 & 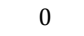 & 0 & 2.053 & 0 \\
\hline & 0 & 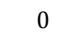 & 0.0124 & 0 & $I_{z} \min _{x} z_{2}$ & 11.2913 \\
\hline & {$[0.012$} & 0 & 0 & 1.2913 & 0 & $-I_{x} \max _{x}$ \\
\hline \multirow[t]{2}{*}{$4=$} & 0 & 0.0023 & 0 & 0 & 2.053 & 0 \\
\hline & 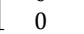 & & 124 & & $\max _{x} z_{2}$ & 1.2913 \\
\hline
\end{tabular}




\subsection{Integral control action}

If the performance of the fuzzy PDC results in unsatisfactory steady-state error, then an integral control action can be introduced into the control loop. Steady-state error is often reduced, if not totally eliminated, by the integral control action, but care must be taken because it raises the system order by one, thus resulting in a higher-order system that tends to oscillate unstably.

\section{Stability analysis}

According to the stability theorem in [11] that follows directly from the Lyapunov stability theory, all 16 of the following linear matrix inequalities (LMIs) must be satisfied by one positive definite symmetric matrix $\mathbf{P}$

$\left(\mathbf{A}_{1}-\mathbf{B K}_{1}\right)^{T} \mathbf{P}+\mathbf{P}\left(\mathbf{A}_{1}-\mathbf{B K}_{1}\right)<\mathbf{0}, \quad\left(\mathbf{A}_{3}-\mathbf{B K}_{1}\right)^{T} \mathbf{P}+\mathbf{P}\left(\mathbf{A}_{3}-\mathbf{B K}_{1}\right)<\mathbf{0}$ $\left(\mathbf{A}_{1}-\mathbf{B K}_{2}\right)^{T} \mathbf{P}+\mathbf{P}\left(\mathbf{A}_{1}-\mathbf{B K}_{2}\right)<\mathbf{0}, \quad\left(\mathbf{A}_{3}-\mathbf{B K}_{2}\right)^{T} \mathbf{P}+\mathbf{P}\left(\mathbf{A}_{3}-\mathbf{B K}_{2}\right)<\mathbf{0}$ $\left(\mathbf{A}_{1}-\mathbf{B K}_{3}\right)^{T} \mathbf{P}+\mathbf{P}\left(\mathbf{A}_{1}-\mathbf{B K}_{3}\right)<\mathbf{0}, \quad\left(\mathbf{A}_{3}-\mathbf{B K}_{3}\right)^{T} \mathbf{P}+\mathbf{P}\left(\mathbf{A}_{3}-\mathbf{B K}_{3}\right)<\mathbf{0}$ $\left(\mathbf{A}_{1}-\mathbf{B K}_{4}\right)^{T} \mathbf{P}+\mathbf{P}\left(\mathbf{A}_{1}-\mathbf{B K}_{4}\right)<\mathbf{0}, \quad\left(\mathbf{A}_{3}-\mathbf{B K}_{4}\right)^{T} \mathbf{P}+\mathbf{P}\left(\mathbf{A}_{3}-\mathbf{B K}_{4}\right)<\mathbf{0}$ $\left(\mathbf{A}_{2}-\mathbf{B K}_{1}\right)^{T} \mathbf{P}+\mathbf{P}\left(\mathbf{A}_{2}-\mathbf{B K}_{1}\right)<\mathbf{0}, \quad\left(\mathbf{A}_{4}-\mathbf{B K}_{1}\right)^{T} \mathbf{P}+\mathbf{P}\left(\mathbf{A}_{4}-\mathbf{B K}_{1}\right)<\mathbf{0}$ $\left(\mathbf{A}_{2}-\mathbf{B K}_{2}\right)^{T} \mathbf{P}+\mathbf{P}\left(\mathbf{A}_{2}-\mathbf{B K}_{2}\right)<\mathbf{0}, \quad\left(\mathbf{A}_{4}-\mathbf{B K}_{2}\right)^{T} \mathbf{P}+\mathbf{P}\left(\mathbf{A}_{4}-\mathbf{B K}_{2}\right)<\mathbf{0}$ $\left(\mathbf{A}_{2}-\mathbf{B K}_{3}\right)^{T} \mathbf{P}+\mathbf{P}\left(\mathbf{A}_{2}-\mathbf{B K}_{3}\right)<\mathbf{0}, \quad\left(\mathbf{A}_{4}-\mathbf{B K}_{3}\right)^{T} \mathbf{P}+\mathbf{P}\left(\mathbf{A}_{4}-\mathbf{B K}_{3}\right)<\mathbf{0}$ $\left(\mathbf{A}_{2}-\mathbf{B K}_{4}\right)^{T} \mathbf{P}+\mathbf{P}\left(\mathbf{A}_{2}-\mathbf{B K}_{4}\right)<\mathbf{0}, \quad\left(\mathbf{A}_{4}-\mathbf{B K}_{4}\right)^{T} \mathbf{P}+\mathbf{P}\left(\mathbf{A}_{4}-\mathbf{B K}_{4}\right)<\mathbf{0}$

Using a numerical LMI solver, it can be verified that the following symmetric matrix $\mathbf{P}$

$\mathbf{P}=10^{8} \cdot\left[\begin{array}{cccccc}0.0000 & -0.0000 & 0.0000 & 0.0003 & 0.0000 & 0.0000 \\ -0.0000 & 0.0000 & -0.0000 & -0.0000 & 0.0042 & -0.0000 \\ 0.0000 & -0.0000 & 0.0000 & 0.0000 & 0.0000 & 0.0003 \\ 0.0003 & -0.0000 & 0.0000 & 1.5520 & -0.0000 & 0.0000 \\ 0.0000 & 0.0042 & 0.0000 & -0.0000 & 2.1548 & -0.0000 \\ 0.0000 & -0.0000 & 0.0003 & 0.0000 & -0.0000 & 1.5520\end{array}\right]$

is positive definite because all its eigenvalues are positive

$\lambda=\left\{144.5244,144.5244,2260.3,1.5520 \cdot 10^{8}, 1.5520 \cdot 10^{8}, 2.1548 \cdot 10^{8}\right\}$

and it satisfies all these 16 LMIs. Therefore, we are guaranteed that the designed fuzzy PDC controller is able to drive the Euler angles to converge to zero in the above closed-loop control system.

\section{Numerical results}

In this section, the effectiveness of the proposed control scheme is demonstrated by numerical simulations. The spacecraft is assumed to travel in a circular orbit with an altitude of $499.075 \mathrm{~km}$, with the orbital period of 5667 seconds and to track the Sun at $360^{\circ}$ per orbit. The simulation settings for the attitude control system are shown on Table 1.

Table 1: Simulation settings for attitude control system

\begin{tabular}{|l|l|}
\hline \multicolumn{1}{|c|}{ Parameters } & \multicolumn{1}{|c|}{ Values } \\
\hline $\begin{array}{l}\text { Spacecraft's } \\
\text { principal moments of } \\
\text { inertia }\left(\mathrm{kg}-\mathrm{m}^{2}\right)\end{array}$ & {$\left[\begin{array}{l}I_{x} \\
I_{y} \\
I_{z}\end{array}\right]=\left[\begin{array}{c}3102 \\
564 \\
3102\end{array}\right]$} \\
\hline $\begin{array}{l}\text { Solar array moment } \\
\text { of inertia }\left(\mathrm{kg}-\mathrm{m}^{2}\right)\end{array}$ & $I_{a}=7.35$ \\
\hline $\begin{array}{l}\text { Orbital velocity } \\
\text { (rad/s) }\end{array}$ & $\omega_{\odot}=0.001108732$ \\
\hline $\begin{array}{l}\text { External } \\
\text { disturbance torques } \\
\text { (Nm) }\end{array}$ & {$\left[\begin{array}{l}T_{d x} \\
T_{d y} \\
T_{d z}\end{array}\right]=\left[\begin{array}{c}2.4 \sin \left(\omega_{\odot} t\right)+1.8 \cos \left(\omega_{\odot} t\right)+6 \\
1.8 \cos \left(\omega_{\odot} t\right)+6\end{array}\right] \cdot 10^{-6}$} \\
\hline $\begin{array}{l}\text { Desired attitude } \\
\text { pointing accuracy }\end{array}$ & $0^{\circ} \pm 0.1^{\circ}$ \\
\hline $\begin{array}{l}\text { Initial attitude of the } \\
\text { spacecraft }\end{array}$ & {$\left[\begin{array}{l}\phi(0) \\
\theta(0) \\
\psi(0)\end{array}\right]=\left[\begin{array}{l}3^{\circ} \\
6^{\circ} \\
9^{\circ}\end{array}\right]$} \\
\hline PDC gains & See Eq. $(24)$ \\
\hline $\begin{array}{l}\text { Integral gain (pitch- } \\
\text { axis only) }\end{array}$ & $k_{i y}=4.0672 \times 10^{-7}$ \\
\hline
\end{tabular}

(a) Transient response of Euler angles under fuzzy PDC only

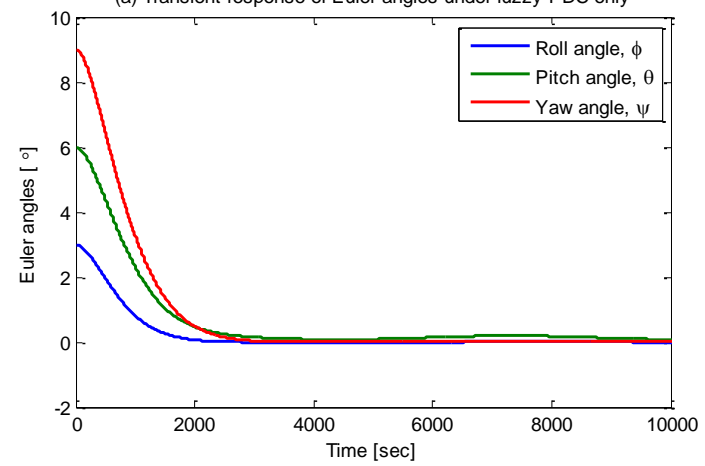

(b) Steady-state response of Euler angles under fuzzy PDC only

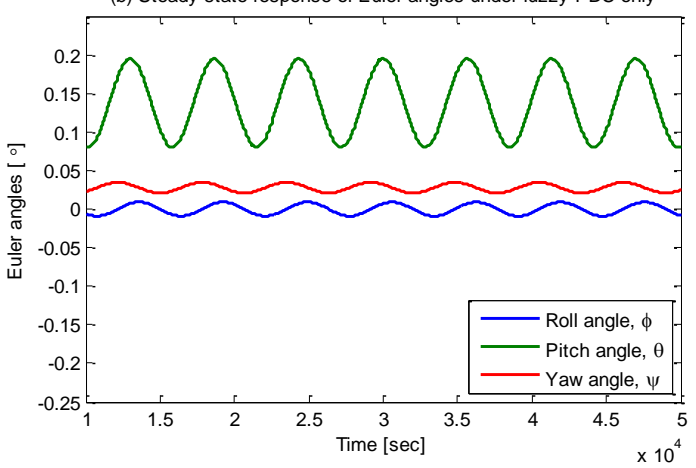

(c) Transient response of Euler angles under PDC with integral action

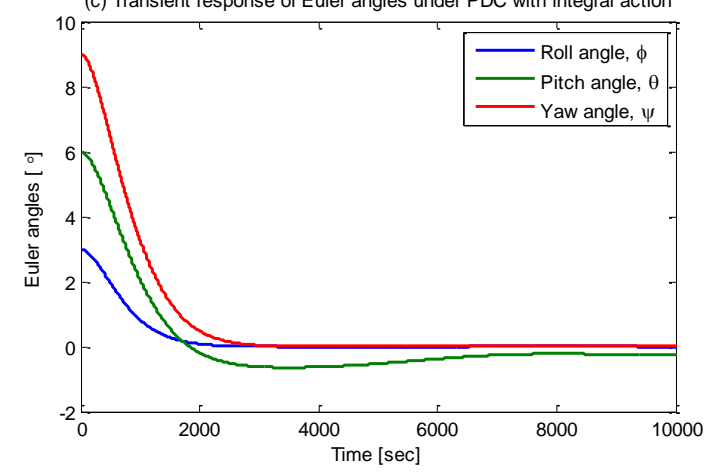

(d) Steady-state response of Euler angles under PDC with integral action

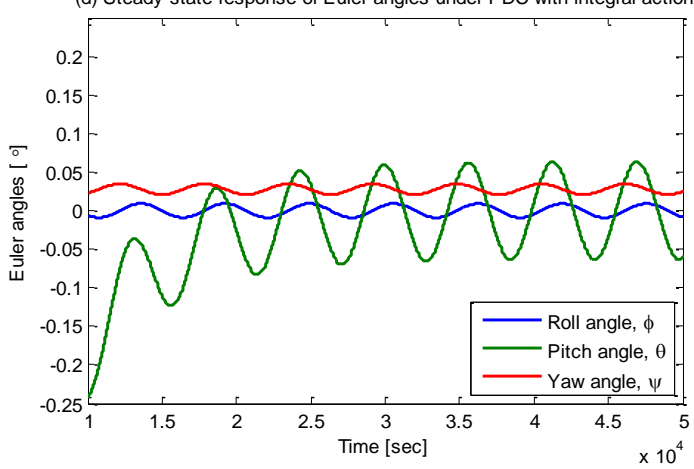

Fig. 3: Time responses of Euler angles under fuzzy PDC only (a \& b) and PDC with integral control action (c \& d).

A set of subplots in Figure 3 show the time responses of the Euler angles, where Figures $3 \mathrm{a}$ and $3 \mathrm{~b}$ are under fuzzy PDC and Figures $3 \mathrm{c}$ and $3 \mathrm{~d}$ are under fuzzy PDC with integral action. For comparison purposes, the subplots are split into transient and steady-state responses. The fuzzy PDC is able to stabilize the spacecraft attitude in the presence of external disturbances as shown in Figures $3 \mathrm{a}$ and $3 \mathrm{c}$, and the results are consistent with the stability analysis. In Figure 3b, however, it is relatively easy to observe that the offset on the pitch attitude at steady-state is fairly significant when compared to the other two Euler angles. If the pitch accuracy is unsatisfactory, the integral action can be introduced to the pitch dynamics to alleviate the offset as shown in Figure 3d. 


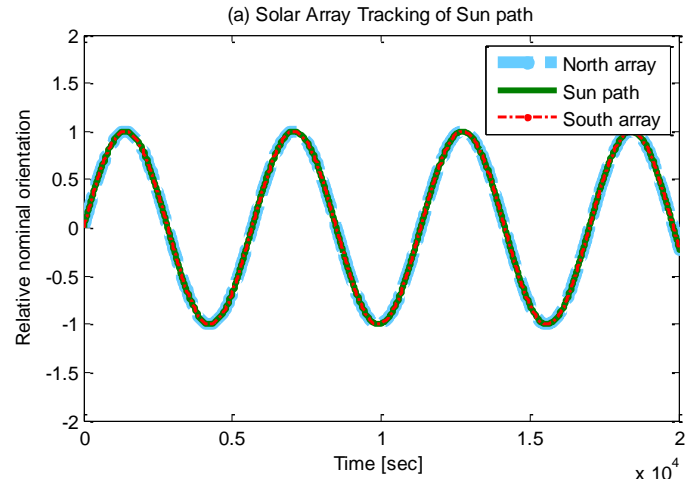

(b) Angle of incidence between incoming sunlight and the arrays

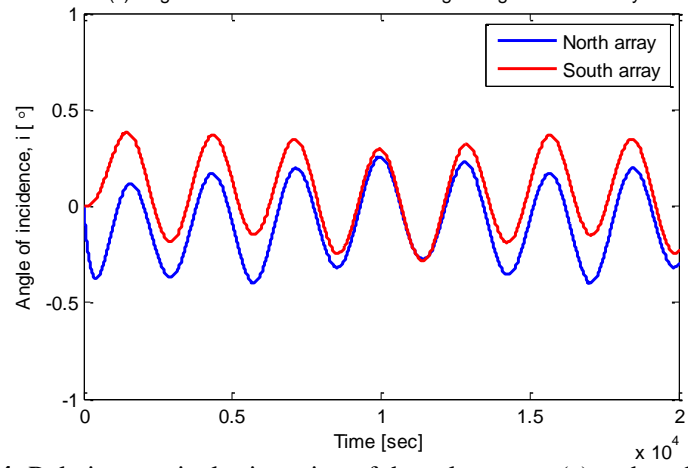

Fig. 4: Relative nominal orientation of the solar arrays (a) and angle of incidence on the solar arrays (b).

On the Sun-tracking performance, both solar arrays are able to track the Sun with a good precision. From the measured angular velocity of the solar array $\omega_{a}$, and computing $\sin \left(\omega_{a} t\right)$ for tracking the apparent trajectory of the Sun, the relative nominal orientations of the solar arrays are plotted on Figure 4a. As expected from Eqs. (3-4), the solar arrays can only follow the Sun's trajectory, but cannot track the Sun perfectly, because the differential torque is constantly generated to stabilize the attitude error, even in the eclipse phase.

Another way to measure the performance of the solar array in Sun-tracking is the angle of incidence between the incoming Sunlight and the solar array. Figure $4 \mathrm{~b}$ shows the angle of incidences are regulated under $0.5^{\circ}$ and this is highly desirable because a $5^{\circ}$ Sun pointing error will cause a drop in the power generation by $0.4 \%$ due to $\cos \left(5^{\circ}\right)=0.996$.

\section{Conclusion}

In this paper, we have designed a fuzzy controller to deal with the spacecraft combined attitude and Sun tracking control problem, which is a nonlinear system, via the parallel distributed compensation approach. The dynamics of a rigid spacecraft is characterized using a Takagi-Sugeno fuzzy model. The main contribution of this paper is about providing a simple yet systematic framework for the modelling, design and stability analysis of a nonlinear spacecraft attitude control system using fuzzy logic. The parallel distributed compensation approach shares the same problemsolving philosophy as the principle of divide and conquer. The stability condition to ensure asymptotic stability is highlighted. Numerical simulations on the spacecraft with CASTS configuration have shown that both the attitude and Sun trajectory tracking capabilities are well achieved using the proposed fuzzy PDC scheme with the integral control action

\section{References}

[1] Berger E (2018), The Zuma failure has emboldened critics of SpaceX. Ars Technica. Available: https://arstechnica.com/science/ 2018/01/the-zuma-failure-has-emboldened-critics-of-spacex/
[2] Robertson B (2003), Stoneking E. Satellite GN \& C Anomaly Trends, Annual AAS Rocky Mountain Guidance and Control Conference, American Astronautical Society; [Online]. Available at: http://ntrs.nasa.gov/search.jsp?R=20030025663

[3] Varatharajoo R (2006), Operation for the combined energy and attitude control system. Aircraft Engineering and Aerospace Technology: An International Journal 78(6): 495-501.

[4] Varatharajoo R \& Kahle R (2005), A review of conventional and synergistic systems for small satellites. Aircraft Engineering and Aerospace Technology: An International Journal 77(2): 131-141.

[5] Chak Y-C \& Varatharajoo R (2015), A novel design of spacecraft combined attitude \& sun tracking system using a versatile fuzzy controller. Aircraft Engineering and Aerospace Technology: An International Journal 87(6): 530-539.

[6] Chak Y-C, Varatharajoo R \& Razoumny Y (2017), Disturbance observer-based fuzzy control for flexible spacecraft combined attitude \& sun tracking system. Acta Astronautica 133: 302-310.

[7] Takagi T \& Sugeno M (1985), Fuzzy identification of systems and its applications to modeling and control. IEEE Transactions on Systems, Man, and Cybernetics. IEEE; SMC-15(1): 116-132.

[8] Tanaka K \& Wang HO (2001), Fuzzy Control Systems Design and Analysis. John Wiley \& Sons.

[9] Lilly JH (2010), Parallel Distributed Control with Takagi-Sugeno Fuzzy Systems. Fuzzy Control and Identification. John Wiley \& Sons.

[10] Sugeno M \& Kang GT (1986), Fuzzy modelling and control of multilayer incinerator. Fuzzy Sets and Systems 18(3): 329-345.

[11] Tanaka K \& Sugeno M (1992), Stability analysis and design of fuzzy control systems. Fuzzy Sets and Systems 45(2):135-156.

[12] Wang HO, Tanaka K \& Griffin M (1995), Parallel distributed compensation of nonlinear systems by Takagi-Sugeno fuzzy model. IEEE International Conference on Fuzzy Systems. 